\title{
Crecimiento, acumulación y distribución de materia seca en dos variedades de amaranto (Amaranthus hypochondriacus y A. cruentus) bajo fertigación
}

\author{
Growth, accumulation and distribution of dry matter in two varieties of amaranth (Amaranthus \\ hypochondriacus L. y A. cruentus L.) under fertigation
}

\begin{abstract}
Diego Monroy-Pedroza', José de Jesús Martínez-Hernández', Francisco Gavi-Reyes², Margarita Torres-Aquino ${ }^{1 *}$, Ismael Hernández-Ríos'

Posgrado Innovación en Manejo de Recursos Naturales, Colegio de Postgraduados, Campus San Luis Potosí. Iturbide No. 73, Colonia Centro. Salinas de Hidalgo, CP 78620, San Luis Potosí, México.

2 Postgrado en Hidrociencias, Colegio de Postgraduados, Campus Montecillo. Km. 36.5 Carretera México-Texcoco. CP 56230, Montecillo, Edo. de México, México.
\end{abstract}

\section{ABSTRACT}

Amaranth is a crop adapted to arid and semi-arid agroclimatic conditions. Its grain yield can be increased with fertigation, but there is limited information on growth parameters and crop yield under this system. The objective of this work was to characterize growth, accumulation and distribution of biomass of two varieties of amaranth (Frondosa and Dorada) with fertigation. The experimental phase was carried out is Salinas, San Luís Potosí, at a density of 25 plants per $\mathrm{m}^{2}$. Organomineral fertilization was applied to soil, and chemical fertilization through irrigation, whose total water sheet was $321 \mathrm{~mm}$. Frondosa compared to Dorada, reached faster maturity and presented higher values of: leaves number, panicle length and dry biomass, as well as harvest index (gain/total dry biomaas). Thermal requirement was $910.7^{\circ} \mathrm{C}$ and $968.6^{\circ} \mathrm{C}$ for Frondosa and Dorada, respectively. Frondosa had higher water use efficiency (WUE) $\left(7.1 \mathrm{~kg} \mathrm{~mm}^{-1}\right)$ in grain production (2.3 $\left.\mathrm{t} \mathrm{ha}^{-1}\right)$, but WUE for fresh biomass $\left(69.6 \mathrm{t} \mathrm{ha}^{-1}\right)$ was higher in Dorada (216.6 kg mm $\mathrm{mm}^{-1}$ ). Environmental conditions in Altiplano Potosino are favorable to produce amaranth with high yield (grain or forage) under fertigation.

Keywords: Amaranthus spp, growth dynamics, yield components, fertigation, water use efficiency

\section{RESUMEN}

El cultivo de amaranto se adapta a condiciones agroclimáticas de regiones áridas y semiáridas. Su rendimiento de grano puede incrementarse con fertirriego, pero es necesario generar más información sobre parámetros de crecimiento y rendimiento del cultivo bajo este sistema. El objetivo fue caracterizar el crecimiento, acumulación y distribución de materia seca de dos variedades de amaranto (Frondosa y Dorada) bajo fertigación. La fase de campo se realizó en Salinas, San Luis Potosí, con una densidad de 25 plantas por $\mathrm{m}^{2}$. Se aplicó fertilizante organomineral al suelo y nutrición química en el agua de riego, cuya lámina total fue $321 \mathrm{~mm}$. Frondosa, respecto a la Dorada, llegó más rápido a madurez y mostró valores mayores en: número de hojas; longitud y biomasa seca de panoja; e índice de cosecha (grano/biomasa total).
El requerimiento térmico total fue de $910.7^{\circ} \mathrm{C}$ y $968.6^{\circ} \mathrm{C}$ para Frondosa y Dorada, respectivamente. Frondosa tuvo mayor eficiencia en el uso del agua (EUA) $\left(7.1 \mathrm{~kg} \mathrm{~mm}^{-1}\right)$ en grano (2.3 $\left.\mathrm{t} \mathrm{ha}^{-1}\right)$, pero la EUA para biomasa fresca $\left(69.6 \mathrm{t} \mathrm{ha}^{-1}\right)$ fue mayor en Dorada ( $216.6 \mathrm{~kg} \mathrm{~mm}^{-1}$ ). Las condiciones ambientales del Altiplano Potosino son favorables para producir Amaranto con alto rendimiento de grano o forraje con fertigación.

Palabras clave: Amaranthus spp, dinámica de crecimiento, componentes de rendimiento, fertigación, eficiencia de uso de agua

\section{INTRODUCCIÓN}

El género Amaranthus L. pertenece a la familia Amaranthaceae e incluye cerca de 75 especies, el 44\% de las cuales son originarias de América y el resto de Australia, África, Asia y Europa (Costea et al., 2001). Algunas especies de este género son malezas; por ejemplo, $A$. spinosus, $A$. tuberculatus y $A$. retroflexus; mientras que las especies, $A$. hypocondriacus, $A$. caudatus y $A$. cruentus tienen un interés agronómico, ya que su grano supera nutrimentalmente al de maíz, arroz y trigo (Paredes-López et al., 2006; Maurya y Arya, 2018). $A$. hypocondriacus y $A$. caudatus son utilizadas con doble propósito (grano y forraje), A. blitum, A. dubius y A. tricolor se aprovechan como verdura por sus propiedades nutrimentales similares a la espinaca (Spinacia oleracea) y la acelga (Beta vulgaris); algunas, por su color, son utilizadas también como plantas de ornato (Akubugwo et al., 2007; Abalone et al., 2004; Arellano et al., 2004; Brenner et al., 2000). Recientemente se ha investigado el potencial de la harina de amaranto como antioxidante y como bebidas funcionales o energéticas, con el fin de promover la salud de los consumidores y para mejorar la resistencia física de deportistas (Almirudis-Echeverría et al., 2020; Argüelles-López et al., 2018; Espino-González et al., 2018).

El rendimiento promedio de grano de amaranto es $1.31 \mathrm{t} \mathrm{ha}^{-1}$ (SIAP, 2017), por lo que el cultivo de esta especie es económicamente viable (Ayala-Garay et al., 2014). En San Luis Potosí, la producción de amaranto inició recientemente (González et al., 2014), y por ello existe poca información 
sobre su dinámica de crecimiento, la cual es importante para calendarizar labores agrícolas apropiadas para el cultivo (Sifuentes et al., 2015).

El crecimiento es el incremento irreversible en el tamaño de las plantas, que está acompañado por los procesos de morfogénesis y diferenciación (Radosevich y Holt, 1984) y puede representarse mediante curvas de crecimiento (Gimplinger y Kaul, 2009). Estos procesos se pueden medir mediante la tasa absoluta de crecimiento, en función de la cantidad de materia seca acumulada y la tasa de funcionamiento de ésta (Goudriaan y Van Laar, 1994) en relación con la influencia del ambiente (Milthorpe y Moorby, 1982).

La acumulación de materia seca es el criterio más utilizado para medir el crecimiento y la magnitud del sistema de asimilación de la planta, el cual es referido frecuentemente al área foliar total (Taiz y Zeiger, 1991). Dicha acumulación varía a través del ciclo de vida del cultivo, por lo que la materia seca y área foliar son muestreadas a intervalos de días o semanas para cuantificar efectos ambientales o analizar diferencias genotípicas entre variedades de cultivos (Shibles, 1987).

Es importante señalar que los fotosintatos pueden usarse para crecimiento vegetativo, síntesis de material de almacenamiento y respiración; asimismo la proporción dirigida hacia estas tres demandas dependen de la etapa fisiológica de la planta. En plantas jóvenes, el crecimiento estructural domina, por lo que más de la mitad de los fotosintatos asimilados son usados para el crecimiento; en plantas maduras, la mayor proporción de fotosintatos son empleados en sintetizar material de almacenamiento; y durante el periodo de formación y llenado de grano o fruto aproximadamente el $80 \%$ de los fotosintatos transportados son usados como metabolitos para estos sitios de demanda (Mengel y Kirkby, 1978).

El amaranto puede desarrollarse en zonas áridas y semiáridas (Schahbazian et al., 2006); sin embargo, su potencial productivo es limitado principalmente por la escasez de agua, ya sea de lluvia o de riego (Ejieji y Adeniran, 2010), situación que puede superarse con la aplicación de tecnologías enfocadas al uso eficiente del agua, tales como el riego por goteo y la fertigación.

La presente investigación tuvo como objetivo estudiar la dinámica de crecimiento, acumulación y distribución de materia seca, así como la producción de forraje y grano en dos variedades de amaranto bajo condiciones de fertigación.

\section{MATERIALES Y MÉTODOS}

El presente trabajo se realizó en la comunidad de La Palma Pegada, municipio de Salinas, San Luis Potosí, en las coordenadas geográficas $22^{\circ} 43^{\prime} 08.0^{\prime \prime} \mathrm{N} 101^{\circ} 48^{\prime} 19.1^{\prime \prime} \mathrm{W}$ y a 2,070 msnm. El suelo utilizado se caracterizó por ser de textura franco-arenosa, $\mathrm{pH}$ alcalino (7.7), conductividad eléctrica baja $\left(0.13 \mathrm{dS} \mathrm{m}^{-1}\right)$, presentar valores de materia orgánica (2.4\%), nitrógeno $(0.12 \%)$, potasio $(46.8 \mathrm{ppm}$ ) y fósforo $(98.8$ ppm), que se clasifican como medio, bajo, bajo y alto, respectivamente (Moreno, 1978).

\section{Material biológico y siembra}

Se utilizó semilla de dos especies de amaranto: $A$. hypochondriacus, var. Frondosa derivada de la raza Mercado (Torres et al., 2006); y A. cruentus, var. Dorada derivada de la raza Mexicana (Maldonado, 2014). La siembra se realizó el 21 de agosto de 2015. Ambas variedades fueron los tratamientos evaluados bajo un diseño experimental de parcelas divididas en bloques completamente al azar, con seis repeticiones. Donde la parcela grande fueron las variedades y la parcela chica las diferentes fechas de muestreo. Cada unidad experimental fue de $5.6 \mathrm{~m}^{2}(7.0 \times 0.8 \mathrm{~m})$ de longitud con camas de cultivo de $40 \times 30 \mathrm{~cm}$ (ancho x profundidad) a 80 $\mathrm{cm}$ de separación entre camas. Cada cama tuvo dos hileras de plantas. La distancia entre éstas fue de $20 \mathrm{~cm}$ y de $10 \mathrm{~cm}$, respectivamente ( 25 plantas $\mathrm{m}^{-2}$ ).

\section{Fertilización de fondo}

Previo a la siembra, se preparó una formula órganomineral la cual consistió en 9.6 de $\mathrm{t} \mathrm{ha}^{-1}$ de estiércol de borrego (previamente cribada) y la fórmula de fertilización mineral 60-60-60 (kg ha-1 de $\mathrm{N}, \mathrm{P}_{2} \mathrm{O}_{5}$ y $\mathrm{K}_{2} \mathrm{O}$, respectivamente), dicha fertilización fue adicionada al suelo a una profundidad aproximada de $30 \mathrm{~cm}$.

\section{Fertigación}

Durante el desarrollo del cultivo se suministró la fórmula de fertilización mineral 64-37-71 en un volumen de agua total de $3210 \mathrm{~m}^{-3} \mathrm{ha}^{-1}$ distribuido en 15 fertiriegos. Esta solución nutritiva tuvo valores de conductividad eléctrica y de $\mathrm{pH}$ de $2.55 \mathrm{dS} \mathrm{m}^{-1}$ y 6.1 , respectivamente.

\section{Muestreos de planta}

Se realizaron cinco muestreos destructivos por variedad, colectando tres plantas en cada una de las seis repeticiones, a los 33, 47, 61, 76, 96 y 103 días después de la emergencia (DDE); los primeros cuatro durante la floración y formación de grano; y el último a madurez fisiológica (cosecha).

\section{Variables}

Se evaluó: altura de planta $(\mathrm{cm})$, número de hojas, área foliar $\left(\mathrm{cm}^{2}\right)$, longitud de panoja $(\mathrm{cm})$, diámetro de tallo (cm); peso fresco y seco de raíz, hoja, tallo, panoja y granos (g planta $\left.{ }^{-1}\right)$, rendimiento de grano $\left(\mathrm{t} \mathrm{ha}^{-1}\right)$ e índice de cosecha $\left(I C=[\text { Rendimiento de grano/Biomasa total }]^{*} 100\right)$ (Reinaudi et al., 2011), de acuerdo con la etapa fenológica.

\section{Análisis estadístico}

Los datos obtenidos de cada variable fueron procesados estadísticamente mediante análisis de varianza (ANOVA), prueba de Tukey 0.05 y regresión. En el ANOVA las fuentes de variación fueron variedades, muestreo e interacción variedades*muestreo. Debido a que no fue significativa esta interacción $(p<0.05)$ y fue consistente la tendencia entre una variedad y otra a lo largo del ciclo, los promedios (por variable) de las variedades fueron comparados en cada muestreo, 
mediante pruebas de Tukey 0.05 (Di Rienzo et al., 2016). Las curvas de crecimiento y sus ecuaciones de regresión se obtuvieron con los programas "Microsoft Excel" (Versión 16) y Curve expert 1.4 (Hyams, 2010).

\section{RESULTADOS Y DISCUSIÓN}

En la Tabla 1 se presentan las temperaturas promedio máximas (Tmáx) y mínimas (Tmín) de los meses de agosto a diciembre en el período de 2000-2011 (12 años), así como las registradas durante el experimento (agosto a diciembre, 2015). Durante el periodo de 12 años, la Tmáx $\left(24.1^{\circ} \mathrm{C}\right)$ fue menor con respecto a $2015\left(28.4^{\circ} \mathrm{C}\right)$. Durante los meses de octubre, noviembre y diciembre de 2015 se registraron Tmín más bajas $\left(6.5,4.5\right.$ y $0.1^{\circ} \mathrm{C}$, respectivamente), con respecto al periodo de 12 años. Sin embargo, estas temperaturas no afectaron irreversiblemente al cultivo; lo que difiere con lo señalado por Nieto (1989) y Cristaudo et al. (2007), quienes establecen una Tmín de $8{ }^{\circ} \mathrm{C}$ para que este cultivo cese su crecimiento y de $4{ }^{\circ} \mathrm{C}$ para que sufra daños fisiológicos.

Tabla 1. Temperaturas máximas y mínimas promedio de agosto a diciembre de los años 2000-2011 y del 2015 en el que se desarrolló el experimento.

Table 1. Average maximum and minimum temperatures for the August-December 2000-2011 period, and along the period of this study.

\begin{tabular}{lcccc}
\hline & \multicolumn{4}{c}{ Temperatura promedio } \\
\cline { 2 - 5 } Mes & \multicolumn{3}{c}{ Máxima } & \multicolumn{3}{c}{ Mínima } \\
\cline { 2 - 5 } & $\mathbf{2 0 0 0 - 2 0 1 1}$ & $\mathbf{2 0 1 5}$ & $\mathbf{2 0 0 0 - 2 0 1 1}$ & $\mathbf{2 0 1 5}$ \\
\cline { 2 - 5 } & \multicolumn{3}{c}{${ }^{\circ} \mathrm{C}$} \\
\hline Agosto & 26.2 & 28.3 & 12.6 & 10.5 \\
Septiembre & 25.1 & 30.4 & 12.0 & 8.2 \\
Octubre & 24.5 & 29.1 & 8.9 & 6.5 \\
Noviembre & 23.0 & 28.0 & 5.1 & 4.5 \\
Diciembre & 21.8 & 26.4 & 2.8 & 0.1 \\
\hline Promedio & 24.1 & 28.4 & 8.3 & 6.0 \\
\hline
\end{tabular}

\section{Dinámica de crecimiento de las dos variedades de ama-} ranto

La duración del ciclo de desarrollo fue de 103 días para A. cruentus y de 96 días para A. hypochondriacus (Tabla 2), lo cual se puede asociar con las condiciones climáticas bajo las cuales se desarrolló el presente estudio, aunque Rivelli et al. (2008) establecen un ciclo de cultivo más largo para $A$. cruentus (115 días) y para $A$. hypochondriacus ( 150 días) en el periodo junio a septiembre, en el que regularmente se registran temperaturas mínimas menos frías.

En altura final (96 DDE), Dorada $(173.8 \mathrm{~cm})$ no se diferenció de Frondosa $(162.2 \mathrm{~cm})$, en contraste con lo registrado a los $75 \mathrm{DDE}$, cuando la primera variedad superó en tamaño a la segunda (Tabla 2). Torres et al. (2006) reportan que Frondosa presenta porte intermedio $(170.0 \mathrm{~cm})$. Sin embargo, Mapes et al. (1995) encontraron una menor altura para esta variedad $(144.1 \mathrm{~cm})$; estos mismos autores encontraron una altura similar $(172.6 \mathrm{~cm})$ a la de este estudio para la variedad Dorada.
La mayor tasa de crecimiento (altura/DDE) se obtuvo a los 33 y $47 \mathrm{DDE}$ con la variedad Frondosa $\left(3.17 \mathrm{~cm} \mathrm{día}^{-1}\right)$ y Dorada (3.77 cm día $\left.{ }^{-1}\right)$, respectivamente (Figura 1); periodo durante el cual la temperatura máxima $\left(30.4^{\circ} \mathrm{C}\right)$ y mínima $(8.9$ ${ }^{\circ} \mathrm{C}$ ) se mantuvieron dentro del rango de crecimiento óptimo propuesto por Cristaudo et al. (2007).

El número total de hojas (Tabla 2) se alcanzó a los 76 DDE y fue diferente entre variedades: 46 y 37 para Frondosa y Dorada, respectivamente. A los $47 \mathrm{DDE}$ se observaron las mayores tasas de crecimiento en altura y en número de hojas en ambas variedades, que a dicha etapa de desarrollo habían acumulado el 48.9 y $46.04 \%$ de su requerimiento térmico total, expresado en unidades calor ${ }^{\circ} \mathrm{C}$ (UC), el cual fue de 910.7 y 968.6 para Frondosa y Dorada, respetivamente (Tabla 2). Estos valores son similares a los reportados por GarcíaPereyra et al. (2007) para A. hypochondriacus (959.0 - 977.3 UC) y A. cruentus (959.5 UC) durante el mismo periodo en que se desarrolló el presente estudio, e inferiores a lo encontrado (1629 UC) en los Valles Altos (Díaz-Ortega et al., 2004). El presente resultado constituye la primera referencia de UC para el cultivo de amaranto en el Altiplano Potosino en el ciclo otoño-invierno bajo condiciones de fertigación.

Los valores más altos de biomasa fresca (forraje fresco) se obtuvieron a los $76 \mathrm{DDE}$, siendo superior $(\mathrm{P}<0.05)$ Dorada (278.4 g planta-1 o $\left.62.6 \mathrm{t} \mathrm{ha}^{-1}\right)$, respecto a Frondosa (201.2 g planta $^{-1}$ o $45.2 \mathrm{t} \mathrm{ha}^{-1}$ ) (Tabla 2). Los resultados obtenidos se encuentran dentro del rango reportado para forraje freso de Amaranthus spp, que va de 10 a $70 \mathrm{t} \mathrm{ha}^{-1}$ en función de la variedad, condiciones ambientales y manejo agronómico (Stordahl et al., 1999; Svirskis, 2003). Con A. hypochondriacus, bajo condiciones de riego para el ciclo primavera-verano, el rendimiento de forraje fresco es de $53 \mathrm{t} \mathrm{ha}^{-1}$ (Chávez-Servín et al., 2017), aunque puede variar entre 22.7 a $72.5 \mathrm{t} \mathrm{ha}^{-1}$ (Pereyra et al., 2004).

El área foliar de $A$. hypochondriacus variedad Frondosa se incrementó de los 33 a 63 DDE (de 1002 a $1827 \mathrm{~cm}^{2}$ planta $^{-1}$ ); posterior a este periodo, disminuyó gradualmente debido a la disminución de hojas ocurrida a la madurez fisiológica, llegando a $325.9 \mathrm{~cm}^{2}$ planta ${ }^{-1}$ al final del ciclo; $A$. cruentus variedad Dorada tuvo la misma tendencia, pero con el valor máximo a los 76 DDE (Figura 2). Los valores obtenidos en $A$. cruentus $\left(1521 \mathrm{~cm}^{2}\right.$ planta-1) durante la etapa de floración coinciden con los encontrados por De la Cruz-Guzmán et al. (2010). Con base en la presente investigación se infiere que $A$. hypochondriacus tuvo mayor capacidad de producir fotoasimilados, y por lo tanto generó mayor rendimiento de grano.

\section{Dinámica de crecimiento y acumulación de materia seca}

La materia seca acumulada al final del ciclo de cultivo no fue diferente $(P<0.05)$ entre Frondosa $\left(46.93\right.$ g planta $\left.^{-1}\right)$ y Dorada (42.53 g planta $^{-1}$ ); sin embargo, su dinámica durante dicho ciclo fue desigual. La mayor tasa de acumulación de materia seca ocurrió durante el periodo de 47 a 61 DDE para Frondosa (1.18 g planta día ${ }^{-1}$ y de 61 a 76 DDE para Dorada (1.09 g planta día $\left.{ }^{-1}\right)$. 
Tabla 2. Evaluación de variables por planta y unidades calor durante el ciclo de cultivo de las variedades de amaranto Frondosa y Dorada. Table 2. Plant variables and heat units measured during the growth cycle of Frondosa and Dorada amaranth varieties.

\begin{tabular}{|c|c|c|c|c|c|c|c|c|}
\hline \multirow{2}{*}{ Variedad } & \multirow{2}{*}{ Variable } & \multirow{2}{*}{ Unidades } & \multicolumn{6}{|c|}{ Días después de la emergencia (DD) } \\
\hline & & & 33 & 47 & 61 & 76 & 96 & 103 \\
\hline Frondosa & \multirow{2}{*}{ Altura } & \multirow{2}{*}{$\mathrm{cm}$} & $58.5 \mathrm{a}$ & $103.0 \mathrm{a}$ & $136.5 \mathrm{a}$ & $144.4 \mathrm{~b}$ & $162.17 \mathrm{a}$ & ----- \\
\hline Dorada & & & $62.1 \mathrm{a}$ & $115.0 \mathrm{a}$ & $152.1 \mathrm{a}$ & $172.2 \mathrm{a}$ & $173.4 \mathrm{a}$ & 173.8 \\
\hline Frondosa & \multirow{2}{*}{ Hojas } & \multirow{2}{*}{ No. } & $15 \mathrm{a}$ & $30 a$ & $40 \mathrm{a}$ & $46 a$ & $12 b$ & \\
\hline Dorada & & & $14 \mathrm{a}$ & $21 \mathrm{~b}$ & $24 b$ & $37 \mathrm{~b}$ & $20 a$ & 10 \\
\hline Unidades calor (UC) & & \multirow{2}{*}{${ }^{\circ} \mathrm{C}$} & 292.5 & 153.5 & 141 & 140 & 183.7 & 57.9 \\
\hline UC acumuladas & & & 292.5 & 446 & 587 & 727 & 910.7 & 968.6 \\
\hline Frondosa & \multirow[t]{2}{*}{ Biomasa } & \multirow{2}{*}{ g } & $50.2 \mathrm{a}$ & $141.2 \mathrm{a}$ & $191.6 \mathrm{a}$ & $201.2 b$ & $160.8 \mathrm{a}$ & --- \\
\hline Dorada & & & $86.8 \mathrm{a}$ & $185.1 \mathrm{a}$ & $258.2 \mathrm{a}$ & $278.4 a$ & 195.8 a & 141.2 \\
\hline
\end{tabular}

Medias con la misma letra en cada columna y variable no son estadísticamente diferentes (Tukey $\mathrm{P}<0.05)$. Means with the same letter are not statistically different (Tukey $\mathrm{P}<0.05$ ).

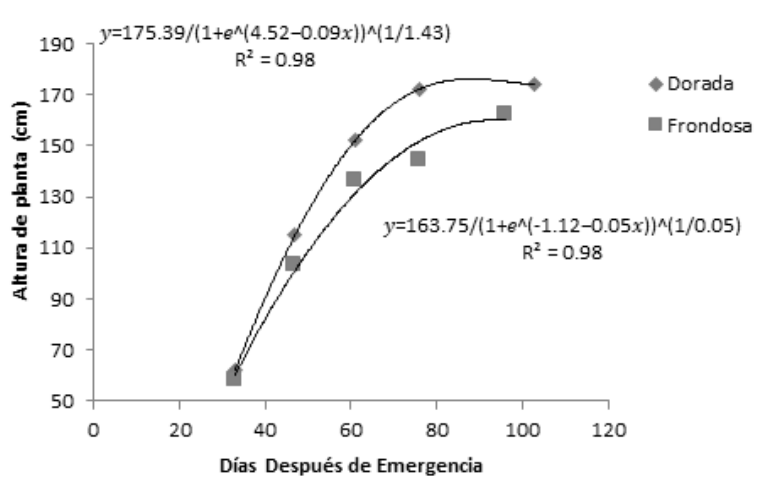

Figura 1. Altura de planta durante el ciclo de cultivo de dos variedades de Amaranto.

Figure 1. Plant height during the growth cycle of two varieties of amaranth.

En general, se espera que la materia seca se acumule en mayor proporción en las estructuras reproductivas (grano, frutos) y en menor cantidad en las estructuras vegetativas; sin embargo, el desarrollo de un cultivo depende de la especie, época del año y condiciones ambientales (Andriolo y Falcão, 2000).

En el presente estudio, la panoja incrementó su longitud linealmente en ambas variedades, pero apareció en menor tiempo (47 DDE) y presentó mayor tasa de elongación $\left(0.38 \mathrm{~cm} \mathrm{día}{ }^{-1}\right)$ en Frondosa respecto a Dorada $\left(0.19 \mathrm{~cm}\right.$ día $\left.{ }^{-1}\right)$ (Figura 3 a). Frondosa también tuvo la panoja más larga, con más peso y mayor producción de grano como se presentará adelante (Tabla 3).

La acumulación de materia seca en hoja y tallo (Figura $3 \mathrm{~b}$ y c) tuvo incrementos decrecientes, que se ajustaron a una ecuación cuadrática, cuyo máximo valor coincidió con la etapa de formación de grano (76 DDE), ya que éste desde su aparición demanda productos para su desarrollo (Vaieretti et al., 2007). En la Figura 3 (b y c) se observa que los valores máximos de acumulación en estas dos variables corresponden a Frondosa, coincidente con mayor rendimiento de biomasa fresca (Tabla 1). Adelante, en acumulación relativa

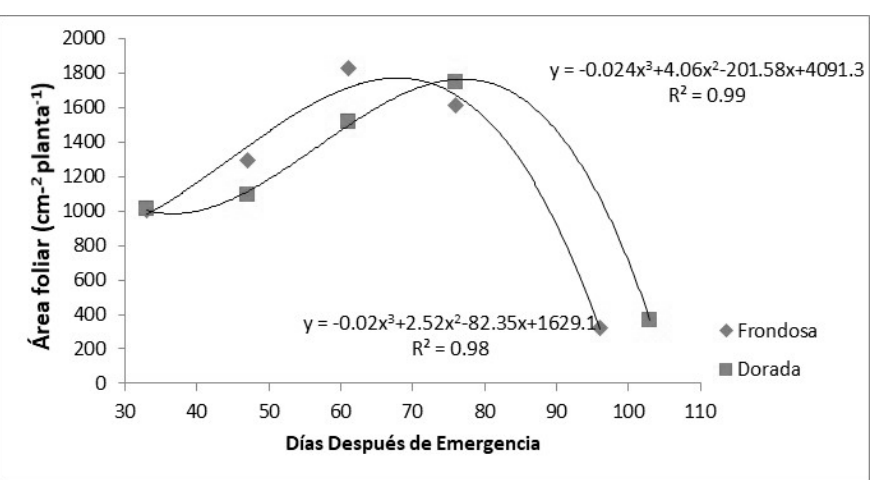

Figura 2. Modelos matemáticos para el área foliar durante el ciclo de cultivo de dos variedades de amaranto.

Figure 2. Mathematical models for leaf area during the growth cycle of two amaranth varieties

de biomasa seca se presentará que la suma de hoja+tallo es mayor $(P<0.05)$ en Dorada, en tanto que el peso seco de la panoja es mayor en Frondosa.

La acumulación de materia seca en la raíz es de tipo sigmoidal y polinómica (cúbica) en Dorada y Frondosa, respectivamente, con incrementos más pequeños en ambos modelos a partir de la floración (Figura 3d), lo cual puede asociarse a la demanda de fotosintatos de la panoja para la formación de grano (Nielsen y Veierskov, 1998). Los modelos mostraron que la raíz tiene un desarrollo en peso similar en ambas variedades desde la emergencia hasta la aparición de la panoja y posteriormente Dorada tuvo una mayor acumulación de biomasa seca.

\section{Partición de biomasa total}

La partición relativa de la biomasa seca, la suma de raíz, tallo, hoja y panoja, se presentan en la Figura 4. En el análisis de los resultados, la mayor asignación de biomasa en la panoja inició desde los 47 DDE en la variedad Frondosa, con un $4.09 \%$ y llegando hasta un $47.13 \%$ a los 96 DDE. Estos valores fueron superiores $(\mathrm{P}<0.05)$ a los cuantificados en la variedad Dorada, en la que la asignación de materia sinteti- 

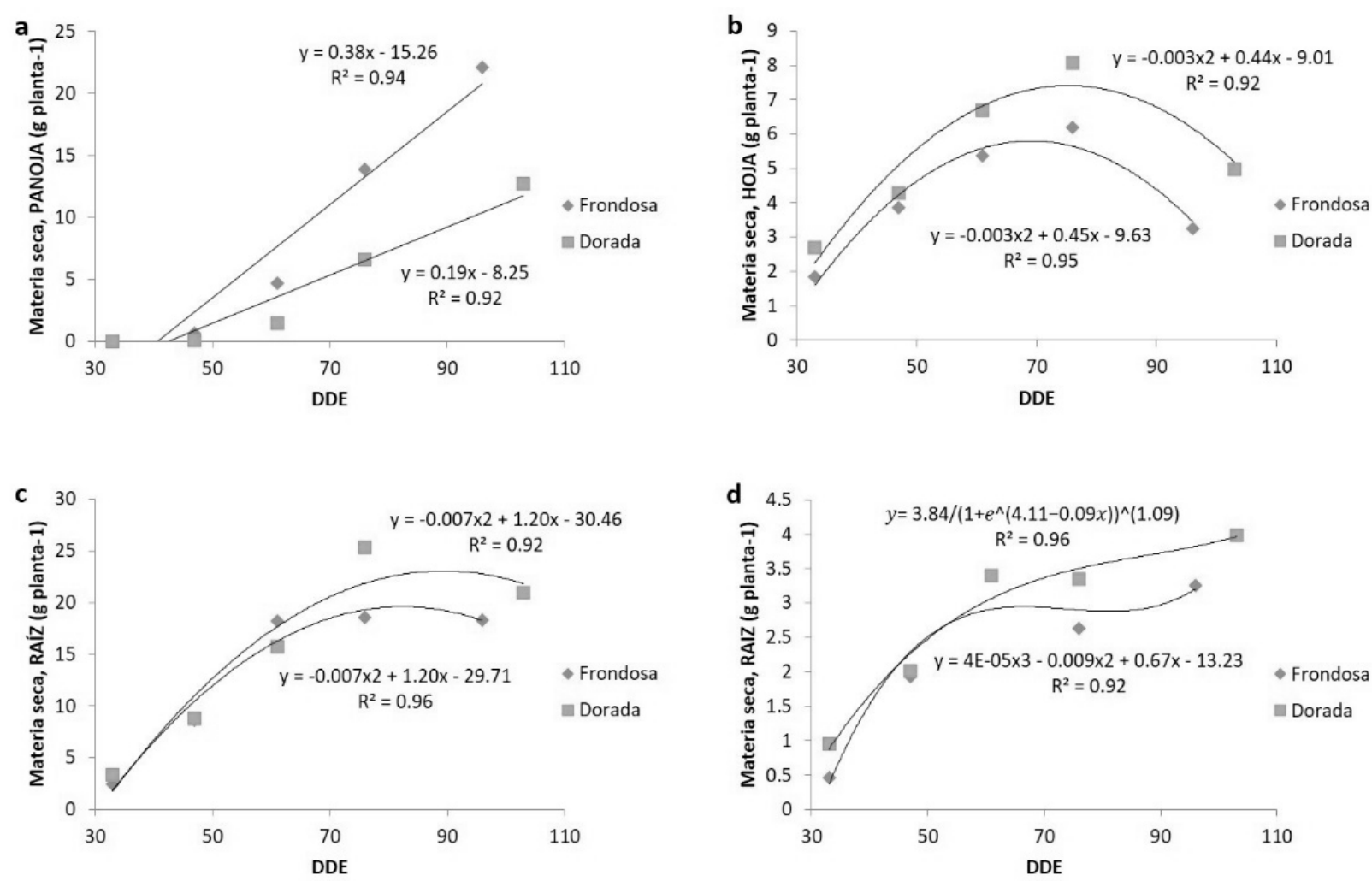

Figura 3. Acumulación de materia seca en función de los días después de la emergencia (DDE): (a) panoja, (b) hoja, (c) tallo y (d) raíz de dos variedades de amaranto.

Figure 3. Dry mass accumulation in panicle (a), leaves (b), stem (c), and root (d) of two amaranth varieties vs days after emergence.

Tabla 3. Longitud y peso final de la panoja, número de granos $\mathrm{g}^{-1}$, rendimiento de grano e índice de cosecha de dos variedades de amaranto. Table 3. Final length and weight of panicle, number of grains $\mathrm{g}^{-1}$, grain yield, and harvest index of two amaranth varieties.

\begin{tabular}{lccccc}
\hline \multirow{2}{*}{ Variedad } & \multicolumn{2}{c}{ Panoja } & Granos por $\mathbf{g}$ & Grano & \multicolumn{2}{c}{ Índice de cosecha } \\
\cline { 2 - 6 } & $\mathbf{c m}$ & $\mathbf{g}$ & No. & t ha $^{-1}$ & \% \\
\hline Frondosa & $39.0 \mathrm{a}$ & $22.1 \mathrm{a}$ & $1131 \mathrm{~b}$ & $2.30 \mathrm{a}$ & $18.0 \mathrm{a}$ \\
Dorada & $33.3 \mathrm{a}$ & $12.7 \mathrm{~b}$ & $1206 \mathrm{a}$ & $1.40 \mathrm{~b}$ & $12.0 \mathrm{~b}$ \\
\hline
\end{tabular}

Medias con la misma letra en cada columna no son estadísticamente diferentes (Tukey $\mathrm{P}<0.05$ ).

Means with the same letter are not statistically different (Tukey $\mathrm{P}<0.05)$.

zada en la misma etapa (47 DDE) sólo presentó un $0.58 \%$ y al final del ciclo (103 DDE) alcanzó un coeficiente de partición de materia seca en panoja de sólo $29.79 \%$.

Asimismo, en ambas variedades se observó que el porcentaje de partición de materia seca de las hojas fue más alto a los 33 DDE (alrededor del 38\%), disminuyendo a medida que se desarrolló el cultivo. Los valores más bajos se tuvieron al final del ciclo de crecimiento $(6.93$ y $11.70 \%$, 96 y $103 \mathrm{DDE}$, Frondosa y Dorada, respectivamente). Lo anterior ocurre debido a que los fotoasimilados están involucrados en la producción foliar y viceversa; conforme trascurre el ciclo de vida de la planta disminuye progresivamente la cantidad de fotoasimilados involucrados en la producción foliar (Vaieretti et al., 2007) y consecuentemente la cantidad de fijación de carbono y la acumulación de materia seca.

En cuanto a tallo, con excepción del porcentaje de partición, a los $96 \mathrm{DDE}$, de la variedad Frondosa (39.1\%), la partición de biomasa seca durante todo el ciclo fue mayor hacia esta estructura, alcanzando valores de hasta $57.63 \%$ y $58.58 \%$ a los 61 DDE en las variedades Frondosa y Dorada; respectivamente. Esto coincide con lo reportado por Rivelli et al. (2008) en especies de amaranto cultivadas en el Mediterráneo en los meses de junio a septiembre.

En ambas variedades se observó que la panoja para su desarrollo demanda fotoasimilados, causando reducción relativa en las demandas de las otras partes de la planta, siendo más significativa en la raíz, que de acuerdo con Nielsen y Veierskov (1998) puede deberse principalmente a la distancia entre el sistema radical y el aparato fotosintético, dando como resultado una baja prioridad en la partición de demanda de fotosintatos; resultado que sugiere que el efecto primario de un incremento en la demanda por causa de la panoja ocasiona una disminución del crecimiento relativo de la raíz; lo cual puede tener relación incluso con una 

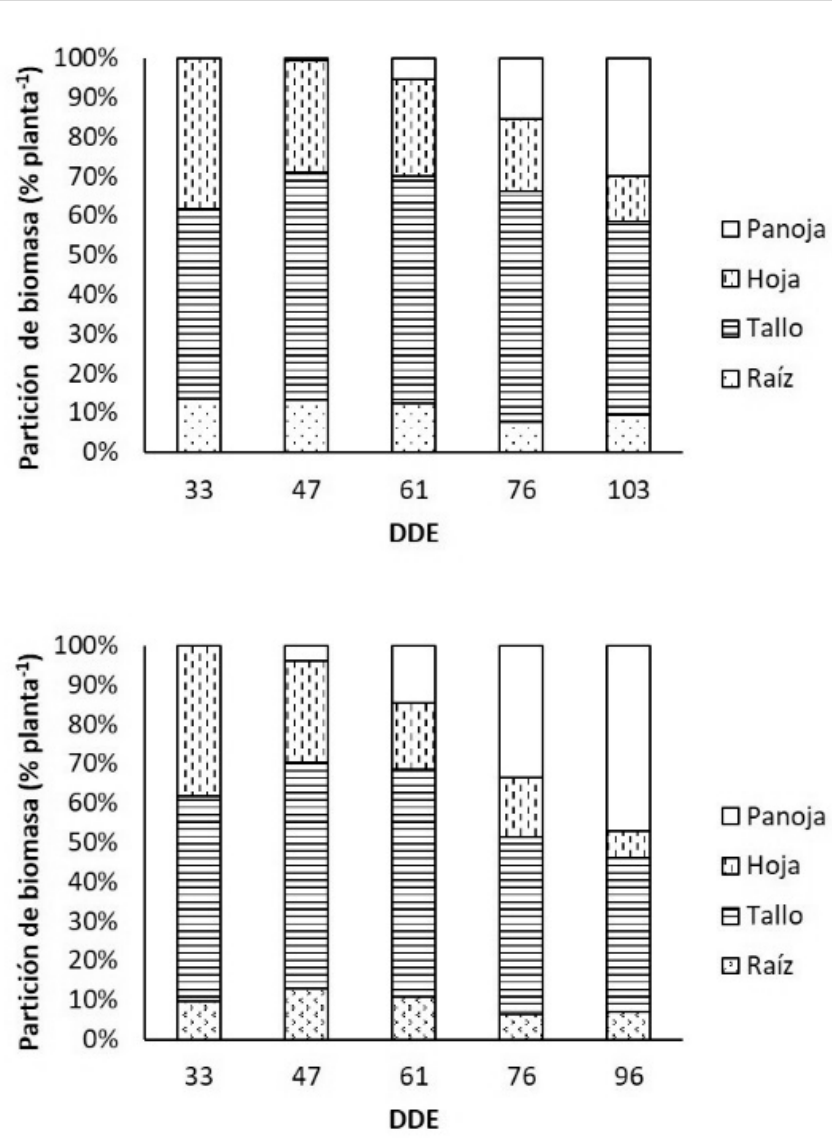

Figura 4. Partición relativa de biomasa en las variedades de amaranto Frondosa (a) y Dorada (b) en función de los días después de la siembra (DDE).

Figure 4. Relative partition of biomass in two amaranth varieties Frondosa (a) Dorada (b) vs days after emergence (DDE).

mayor eficiencia energética ejercida por la planta, dirigida a la mayor producción de frutos y semillas para asegurar la reproducción.

Al considerar sólo las partes reproductivas (panoja) y vegetativa (hoja y tallo) de la planta, se observó que a lo largo del ciclo Frondosa aporta mayor acumulación de biomasa a su estructura reproductiva, logrando a los $96 \mathrm{DDE}$ una acumulación relativa de $47.13 \%$, respecto a los $45.94 \%$ que acumula en la parte vegetativa en el mismo momento. En tanto, Dorada muestra un comportamiento diferente, presentando una acumulación de $60.85 \%$ en la parte vegetativa a los 103 DDE, comparado con $29.79 \%$ en panoja. Este comportamiento coincide por lo reportado en otros trabajos (Becerra, 2000; Morales et al., 2014), donde se menciona que A. cruentus y A. hypochondriacus son especies productoras de grano, siendo que las variedades derivadas de la primera generalmente presentan tendencia a generar follaje, en tanto que las variedades derivadas de la segunda concentran su producción en grano.

\section{Componentes del rendimiento del grano}

En la Tabla 3 se presentan los componentes del rendimiento de las variedades estudiadas. A la cosecha, en longitud de panoja no existió diferencia $(P<0.05)$ entre am- bas variedades, con valores de 39 y $33 \mathrm{~cm}$ para Frondosa y Dorada, respectivamente, que son inferiores a los reportados por Díaz-Ortega et al. (2004) $(61 \mathrm{~cm})$. El peso de la panoja de Frondosa fue significativamente superior al de Dorada (23.8 vs $14.0 \mathrm{~g} \mathrm{planta-1}^{-1}$ y con menor número de granos $\mathrm{g}^{-1}$ (1131 vs $1206 \mathrm{~g}_{\text {por planta-1 }}$ ). El rendimiento de grano fue más alto en Frondosa $\left(2.3 \mathrm{t} \mathrm{ha}^{-1}\right)$ que en Dorada $\left(1.4 \mathrm{t} \mathrm{ha}^{-1}\right)$ lo cual está relacionado con el peso promedio de granos. SIAP (2016) reporta para Amaranthus spp bajo riego rendimientos de grano de 2.13 y $0.9 \mathrm{t} \mathrm{ha}^{-1}$, para los ciclos Primavera-Verano y Otoño-Invierno, respectivamente. En la presente investigación se obtuvieron valores superiores en $155 \%$ y $55 \%$ para Frondosa y Dorada, respectivamente, lo cual se atribuye al uso de Fertirriego. Díaz-Ortega et al. (2004) reportan un menor rendimiento para la variedad Frondosa $\left(1.8 \mathrm{t} \mathrm{ha}^{-1}\right)$ en el ciclo primavera-verano, utilizando mayor volumen de agua y fertilización, con la misma densidad de población que la utilizada en el presente estudio. Chávez-Servín et al. (2017) obtuvieron un rendimiento de $1.4 \mathrm{t} \mathrm{ha}^{-1}$ con 150000 plantas $\mathrm{ha}^{-1}$ en el ciclo primavera-verano bajo condiciones de riego.

El índice de cosecha $(I C)$ fue superior $(P<0.05)$ en la variedad Frondosa (18.0\%) con respecto a la variedad Dorada (12.0\%). Estos valores se encuentran en el rango encontrado en la literatura de amaranto: $11.4 \%$ para raza azteca (DíazOrtega et al., 2004) y $10.5-22.5 \%$ en especies domésticas (Reinaudi et al., 2011).

\section{Uso eficiente del agua}

En referencia a la eficiencia en el uso del agua (EUA), considerando una lámina de $321.4 \mathrm{~mm}$ en el ciclo, ésta fue mayor $(\mathrm{P}<0.05)$ en la producción de biomasa fresca en la variedad Dorada $216.6 \mathrm{~kg} \mathrm{~mm}^{-1}$ con respecto a Frondosa (156.5 $\left.\mathrm{kg} \mathrm{mm}^{-1}\right)$; para la producción de grano, la EUA fue mayor $(P<0.05)$ en la variedad Frondosa $\left(7.15 \mathrm{~kg} \mathrm{~mm}^{-1}\right)$ que en Dorada (4.35 $\left.\mathrm{kg} \mathrm{mm}^{-1}\right)$. En grano, la EUA de ambas variedades fue mayor al reportado por Díaz-Ortega et al. (2004).

La EUA de la variedad para forraje y grano, respectivamente, confirma el uso primordial que puede darse a cada una de las variedades, producción de grano o forraje, bajo las condiciones en que se desarrollaron.

\section{CONCLUSIONES}

Durante la etapa vegetativa, a los $47 \mathrm{DDE}$, la acumulación de UC del amaranto fue: $49 \%$ de $910.7{ }^{\circ} \mathrm{C}$ (Frondosa) y $46 \%$ de $978.6^{\circ} \mathrm{C}$ (Dorada). Estos resultados son la primera referencia para dicha planta en el Altiplano Potosino. La variedad Dorada, comparada con la Frondosa, presentó: crecimiento más rápido; mayor altura (173.8 vs $162.2 \mathrm{~cm}$ ); mayor biomasa fresca total (278.4 vs $201.2 \mathrm{~g}$ planta-1) y seca de raíz. Frondosa alcanzó la máxima área foliar en menor tiempo. La variedad Frondosa, respecto a la Dorada, mostró: mayor número de hojas (46 vs 37) y longitud de panoja (29 vs $33.3 \mathrm{~cm}$ ); mayor biomasa seca en panoja (22.1 vs $12.7 \mathrm{~g}$ ), tallo y hojas; formación de panoja en menor tiempo; mayores Índice de cosecha ( 18 vs $12 \%$ ) y rendimiento ( 2.3 vs $1.4 \mathrm{t} \mathrm{ha}^{-1}$ ). 
En rendimiento de grano, la EUA fue mayor en Frondosa, respecto a Dorada (7.15 vs $\left.4.35 \mathrm{~kg} \mathrm{~mm}^{-1}\right)$. En producción de forraje, la EUA fue mayor en Dorada, respecto a Frondosa (216.6 vs $156.5 \mathrm{~kg} \mathrm{~mm}^{-1}$ ). Las condiciones ambientales del Altiplano Potosino son favorables para producir amaranto para grano (Frondosa) y forraje (Dorada) con fertigación.

\section{REFERENCIAS}

Abalone, R., Cassinera, A., Gastón, A. y Lara, M.A. 2004. Some Physical Properties of Amaranth Seeds. Biosystems Engineering. 89: 109-117.

Akubugwo, I. E., Obasi, N. A., Chinyere, G. C. y Ugbogu, A. E. 2007. Nutritional and chemical value of Amaranthus hybridus L. leaves from Afikpo, Nigeria. African Journal of Biotechnology. 6: 2833-2839.

Almirudis-Echeverria, S. J., Ramírez, W. B., Medina, R. C. L. Magaña, B. E., Torres, Ch. P. I., y Ledesma, O. A.I. 2018. Actividad antioxidante de harinas de amaranto obtenidas por extrusión y análisis parcial de su calidad proteica in vivo. Biotecnia. 22 (1): 24-31.

Andriolo, J.L. y Falcão, L.L. 2000. Efeito da poda de folhas sobre a acumulção de materia seca e sua repartição para frutos do tomateriro cultivado em ambiente protegido. Revista Brasileira de Agrometeorolgia. 8:75-83.

Arellano, M.A., Albarracín, G., Arce, S. y Mucciarelli, S. 2004. Estudio comparativo de hojas de Beta vulgaris con Amaranthus dubuis Mart ex Thell. Phyton. 53: 193-197.

Argüelles-López, O.D., Reyes-Moreno, C., Gutiérrez-Dorado, R., Sánchez-Osuna, M. F., López-Cervantes, J., CuevasRodríguez, E. O., Milán-Carrillo, J., Perales-Sánchez, J. X. K. 2018. Functional beverages elaborated from amaranth and chia flours processed by germination and extrusión. Biotecnia 20 (3):135-45.

Ayala-Garay, A.V., Rivas Valencia, P., Cortes Espinoza, L., de la O Olán, M., Escobedo López, D. y Espitia Rangel, E. 2014. La rentabilidad del cultivo de amaranto (Amaranthus spp.) en la región centro de México. Ciencia Ergo Sum. 21:47-54.

Becerra, R. 2000. El amaranto: nuevas tecnologías para un antiguo cultivo. CONABIO. Biodiversitas. 30: 1-6.

Brenner, D.M., Baltensperger, D.D., Kulakow, P.A., Lehmann, J.W., Myers, R.L., Slabbert, M.M. y Sleugh, B.B. 2000. Genetic resources and breeding of Amaranthus. Plant Breeding Reviews. 19: 227-285.

Chávez-Servín, J.L., Cabrera Baeza, H.F., Jiménez Ugalde, E.A., Mercado Luna, A., de la Torre-Carbot, K., Escobar García, K., Barreyro, A., Serrano Arellano, J. y García-Gasca, T. 2017. Comparison of Chemical Composition and Growth of Amaranth (Amaranthus hypochondriacus) between Greenhouse and Open Field Systems. International Journal of Agriculture and Biology. 19: 577-583.

Costea, M., Sanders, A. y Waines, G. 2001. Preliminary results towards a revision of the Amaranthus hybridus complex (Amaranthaceae). Sida, Contributions to Botany. 19: 931974.

Cristaudo, A., Gresta, F., Luciani, F. y Restuccia, A. 2007. Effects of after-harvest period and environmental factors on seed dormancy of Amaranthus species. Weed Research. 47: $327-$ 334.

De la Cruz-Guzmán, G.H., Arriaga Frías A., Mandujano Piña M. y González Moreno S. 2010. Efecto de la sequía sobre algunas variables hídricas y morfométricas en cinco genotipos de Amaranthus. Idesia (Arica). 28: 87-95.

Díaz-Ortega, A.C., Escalante Estrada, J.A., Trinidad Santos, A., Sánchez García, P., Mapes Sánchez, C. y Martínez Moreno, D. 2004. Rendimiento, eficiencia agronómica del nitrógeno y eficiencia en el uso del agua en amaranto en función del manejo del cultivo. Terra Latinoamericana. 22: 109-116.

Di Rienzo, J.A., Casanoves, F., Balzarini, M.G., Gonzalez, L., Tablada, M. y Robledo, C.W. 2016. InfoStat. Grupo InfoStat, FCA, Universidad Nacional de Córdoba, Argentina.

Ejieji, C.J. y Adeniran, K.A. 2010. Effects of water and fertilizer stress on the yield, fresh and dry matter production of grain amaranth (Amaranthus cruentus L.). Australian Journal of Agricultural Engineering. 1:18-24.

Espino-González, E., Muñoz-Daw, M.J., Rivera-Sosa, J. M., De la Torre-Díaz, M. L., Cano-Olivas, G. E., De Lara-Gallegos, J. C., y Enríquez-Leal, M. C. 2018. The influence of an amaranthbased beverage on cycling performance: a pilot study. Biotecnia. 20 (2): 31-36.

García-Pereyra, J., Aviña Martínez, G.N., Rubio Graciano, R.B., Medina Medrano, R., Alvarado Gómez, O. y Alejandre Iturbide, G. 2007. Requerimiento de unidades calor en el cultivo del amaranto. Revista Biológico Agropecuaria Tuxpan. 5: 1411-1421.

Gimplinger, D., \& Kaul, H. 2009. Calibration and validation of the crop growth model LINTUL for grain amaranth (Amaranthus sp.). Journal of applied botany and food quality. 82: 183-192.

González, A.F.R., Vera, G.A. y Lerma, A.D.L. 2014. Estrategia de comercialización competitiva del amaranto en San Luis Potosí. PERSPECTIVAS. Revista de Análisis de Economía, Comercio y Negocios Internacionales. 10: 71-93.

Goudriaan, J.Y.y Van Laar, H.H. 1994. Modelling potential growth processes. Textbook with exercises. Kluwer Academic Publishers. Springer. Heidelberg Germany.

Hyams, D. 2010. Curve Expert software. Disponible en: https:// docs.curveexpert.net/curveexpert/basicv1/_static/ CurveExpertBasic.pdf.

Maldonado, E. 2014. Análisis proteínico del grano de amaranto (Amaranthus hypochondriacus y A. cruentus) y obtención del mapa proteómico de Amaranthus cruentus. Tesis Doctorado, IPICYT.

Mapes, C., Díaz, A., Collazo, M. y Bye Robert. 1995. Desarrollo de cinco razas de amaranto (Amaranthus spp.) en Chalco, Estado de México. Anales Instituto de Biología. Universidad Nacional Autónoma de México. Serie Botánica. 66: 149-169.

Mengel, K. y Kirkby, E.A. 1978. Principles of plant nutrition. International Potash Institute. Worblaufen-Bern, Switzerland.

Milthorpe, F.L. y Moorby, J. 1982. An Introduction to Crop Physiology. Cambridge University Press. UK.

Morales, J., Vázquez, N. y Bressani, R. 2014. El amaranto Características y aporte nutricio. 2a ed. Trillas. México.

Maurya, N.K. \& Arya, D.P. 2018. Amaranthus grain nutritional benefits: A review. Journal of Pharmacognosy and Phytochemistry. 7(2):2258-2262.

Nielsen, T.H. y Veierskov B, 1998. Distribution of dry matter in sweet pepper plants (Capsicum annuum L.) during the juvenile and generative growth phases. Scientia Horticulturae. 35:179-187.

Nieto, C. 1989. El cultivo de amaranto (Amaranthus spp): Una alternativa agronómica para Ecuador. INIAP 52-28. Estación Experimental Santa Catalina. Quito. 
Paredes-López, O., Guevara- Lara, F. y Bello-Pérez, A. 2006. Los alimentos mágicos de las culturas indígenas mesoamericanas. 1a ed. Ed. Fondo de Cultura Económica. México.

Pereyra, G.J., Valdés, L.C.G.S., Olivares, S.E., Alvarado, G.O., Medrano, R.H. y Alejandre, I. G. 2004. Evaluación de variedades de amaranto para adaptabilidad productiva en el noreste de México. Revista Fitotecnia Mexicana. 27: 53-56.

Radosevich, S.R. y Holt, J.1984. Weed ecology, implications for vegetation management. Jhon Willey and Sons. New York.

Reinaudi, N.B., Repollo, R., Janovská, D., Délamo Frier, J. y Martín de Troiani, R. 2011. Evaluación de genotipos de amaranto (Amaranthus spp.) para la adaptabilidad productiva en el área de la Facultad de Agronomía, Universidad Nacional de la Pampa, Argentina. Revista Científica UDO Agrícola. 11: 50-57.

Rivelli, A.R., Gherbin, P., De Maria, S. y Pizza, S. 2008. Field evaluation of Amaranthus species for seed and biomass yields in Southern Italy. Italian Journal of Agronomy. 3: 2259.

Schahbazian, N., Kamkar, B., Iran-Nejad H. 2006. Evaluation of Amaranth Production Possibility in arid and semi arid Regions of Iran. Asian J. of Plant Scie. 5: 580-585.

SIAP. 2017. Atlas agroalimentario. Servicio de Información Agroalimentaria y Pesquera (SIAP). Disponible en: http:// online.pubhtml5.com/clsi/ibhs/\#p=4.
SIAP. 2016. Acciones y Programas - Producción Agrícola. Servicio de Información Agroalimentaria y Pesquera (SIAP). Disponible en: https://www.gob.mx/siap/acciones-yprogramas/produccion-agricola-33119.

Shibles, R. 1987. Crop physiology. lowa State University. lowa, IA, U.S.A. 214 pp.

Sifuentes, E., Ruelas, J.R., Cervantes, J., Castorena, I., Mondaca, C.A. y López, B.E. 2015. Fenología y tiempo en el manejo del riego y fertilización del cultivo de papa. Biotecnia. 17: 42-48.

Stordahl, J.L., Sheaffer C.C. y Di Costanzo A. 1999. Variety and maturity affect amaranth forage yield and quality. Journal of Production Agriculture. 12:249-253.

Svirskis, A. 2003. Investigation of amaranth cultivation and utilization in Lithuania. Agronomy Research. 1: 253-264.

Taiz, L. y Zeiger, E. 1991. Plant physiology: Mineral nutrition. The Benjamin Cummings Publishing Co., Inc. Redwood City.

Torres, S.G., Trinidad, S.A., Reyna, T.T., Castillo, J.H., Escalante, E.A. y León, G.F. 2006. Respuesta de variedades de amaranto a densidades de población. Revista Fitotecnia Mexicana. 29: 307-312.

Vaieretti, M.V., Diaz, S., Ville, E. y Garnier, E. 2007. Two measurement methods of leaf dry matter content produce similar results in a broad range of species. Annals of Botany. 99: 955-958. 